\title{
Construction of Fourier expansion of Apostol Frobenius-Euler polynomials and its applications
}

\author{
Serkan Araci ${ }^{* *}$ and Mehmet Acikgoz ${ }^{2}$
}

\section{"Correspondence:}

mtsrkn@hotmail.com

'Department of Economics, Faculty

of Economics, Administrative and

Social Science, Hasan Kalyoncu

University, Gaziantep, Turkey

Full list of author information is

available at the end of the article

\begin{abstract}
In the present paper, we find the Fourier expansion of the Apostol Frobenius-Euler polynomials. By using a Fourier expansion of the Apostol Frobenius-Euler polynomials, we derive some new and interesting results.
\end{abstract}

MSC: Primary 11B68; 11S80; secondary 05A19; 42B05

Keywords: Fourier series; Apostol Frobenius-Euler polynomials; Generating function; Lipschitz summation formula; Hurwitz zeta type function

\section{Introduction}

When Fourier was trying to solve a problem in heat conduction, he needed to express a function $f$ as an infinite series of sine and cosine functions:

$$
f(x)=\frac{a_{0}}{2}+\sum_{n=1}^{\infty}\left(a_{n} \cos n x+b_{n} \sin n x\right) .
$$

Earlier, Bernoulli and Euler had used such series while investigating problems concerning vibrating strings and astronomy. Note that a Fourier series is widely known as an expansion of a periodic function $f(x)$ in terms of an infinite sum of sine and cosine functions. Fourier series make use of the orthogonality relationships of the sine and cosine functions.

Fourier series of a function with period $T$ can be written in an exponential form as follows:

$$
\begin{aligned}
f(x)= & a_{0}+a_{1} e^{i w x}+a_{2} e^{2 i w x}+\cdots+a_{n} e^{n i w x}+\cdots \\
& +a_{-1} e^{-i w x}+a_{-2} e^{-2 i w x}+\cdots+a_{-n} e^{-n i w x}+\cdots
\end{aligned}
$$

or equivalently by

$$
f(x)=\sum_{n=-\infty}^{\infty} a_{n} e^{i n w x} \quad\left(w=\frac{2 \pi}{T}\right),
$$

(c) The Author(s) 2018. This article is distributed under the terms of the Creative Commons Attribution 4.0 International License (http://creativecommons.org/licenses/by/4.0/), which permits unrestricted use, distribution, and reproduction in any medium, provided you give appropriate credit to the original author(s) and the source, provide a link to the Creative Commons license, and indicate if changes were made. 
where the coefficients $a_{n}$ and $a_{-n}$ are computed by

$$
a_{n}=\frac{1}{T} \int_{0}^{\frac{2 \pi}{w}} e^{-i n w t} f(t) d t \quad \text { and } \quad a_{-n}=\frac{1}{T} \int_{0}^{\frac{2 \pi}{w}} e^{i n w t} f(t) d t
$$

Note that $a_{-n}=\overline{a_{n}}\left(\overline{a_{n}}\right.$ is the complex conjugate of $\left.a_{n}\right)$. For more information as regards Fourier series, see Refs. $[3,4,6]$.

The Fourier expansion of some well-known polynomials have been studied by some mathematicians; see, for details, [2-4, 6]. For example, in [3], Luo derived a Fourier series and integral representations for the classical Genocchi polynomials, and ApostolGenocchi polynomials by using the Lipschitz summation formula. In [4], by making use of Cauchy residue theorem in the complex plane, Bayad obtained a Fourier series for the Apostol-Bernoulli, Apostol-Genocchi and Apostol-Euler polynomials. Also the Fourier series of sums of products of some well-known special polynomials have been investigated extensively by Agarwal et al. [1] and Kim et al. [6-8, 11].

With this motivation, we are going to focus on obtaining the Fourier expansion of the Apostol Frobenius-Euler polynomial. After that, we are going to derive some useful results arising from its Fourier expansion. Before giving the main results mentioned above, we need some useful properties of Apostol-Frobenius Euler polynomials, which will be given in the next section as preliminaries.

\section{Preliminaries}

The Frobenius-Euler polynomials and their various generalizations such as the Apostol Frobenius-Euler polynomials have been studied intensively by some mathematicians. For example, Kim [2] obtained linear differential equations for Frobenius-Euler polynomials by using their generating function. From those differential equations, he gave the sums of products of Frobenius-Euler polynomials.

We now begin some known definitions and properties of Frobenius-Euler polynomials and Apostol Frobenius-Euler polynomials which will be useful in deriving the main results of this paper.

Definition 1 Let $u \in \mathbb{C}$ with $u \neq 1$. The Frobenius-Euler polynomials $H_{n}(x, u)$ are known as $[2,5]$ :

$$
\sum_{n=0}^{\infty} H_{n}(x, u) \frac{t^{n}}{n !}=\frac{1-u}{e^{t}-u} e^{x t}
$$

Definition 2 Let $u, \lambda \in \mathbb{C}$ with $u \neq 1, \lambda \neq 1$ and $u \neq \lambda$. The Apostol Frobenius-Euler polynomials $H_{n}(x, u, \lambda)$ are defined by $[2,5]$

$$
\sum_{n=0}^{\infty} H_{n}(x, u, \lambda) \frac{t^{n}}{n !}=\frac{1-u}{\lambda e^{t}-u} e^{x t}
$$

Note that Apostol Frobenius-Euler polynomials are a good generalization (or known as $\lambda$ extension) of Frobenius-Euler polynomials. Comparing Definition 1 with Definition 2, one may get the following:

$$
H_{n}(x, u, \lambda)=\frac{u}{\lambda} \frac{1-u}{\lambda} H_{n}\left(x, \lambda u^{-1}\right) .
$$


Observe that

$$
H_{n}(0, u):=H_{n}(u) \quad \text { and } \quad H_{n}(0, u, \lambda):=H_{n}(u, \lambda)
$$

which are called the Frobenius-Euler numbers and Apostol Frobenius-Euler numbers, respectively.

Definition 3 The Frobenius-Genocchi polynomials are defined by means of the following generating function:

$$
\sum_{n=0}^{\infty} G_{n}^{F}(x, u) \frac{t^{n}}{n !}=\frac{(1-u) t}{e^{t}-u} e^{x t} \quad \text { cf. [12]. }
$$

The Apostol-Euler polynomials are defined by means of the following generating series:

$$
\sum_{n=0}^{\infty} E_{n}(x, \lambda) \frac{t^{n}}{n !}=\frac{2}{\lambda e^{t}+1} e^{x t} \quad(|t|<|\log (-\lambda)|)
$$

From Eq. (2), we have

$$
\frac{2}{\lambda e^{t}+1} e^{x t}=\frac{2 \lambda^{-1}}{e^{t}+\lambda^{-1}} e^{x t}=\frac{2}{1+\lambda} \frac{1+\lambda^{-1}}{e^{t}+\lambda^{-1}} e^{x t}=\sum_{n=0}^{\infty} \frac{2}{1+\lambda} H_{n}\left(x, \lambda^{-1}\right) \frac{t^{n}}{n !},
$$

from which one may get the following useful corollary.

\section{Corollary 1}

$$
E_{n}(x, \lambda)=\frac{2}{1+\lambda} H_{n}\left(x, \lambda^{-1}\right), \quad c f .[9] .
$$

Remark 1 Substituting $\lambda=1$ in Definition 2, one can easily see that

$$
H(x, u, 1):=H(x, u), \quad \text { cf. }[2,5] .
$$

Remark 2 Putting $u=-1$ in Definition 2,

$$
H(x,-1, \lambda):=E_{n}(x, \lambda)
$$

where $E_{n}(x, \lambda)$ is called the Apostol-Euler polynomials, cf. [2, 5].

Remark 3 Putting $u=-1$ in Definition 2,

$$
H(x,-1, \lambda):=\frac{G_{n+1}(x, \lambda)}{n+1},
$$

where $G_{n}(x, \lambda)$ is called the Apostol-Genocchi polynomials, cf. [2, 5]. 
Remark 4 Taking $u=-1$ and $\lambda=1$ in Definition 2, one can see

$$
H(x,-1,1):=E_{n}(x)
$$

where $E_{n}(x)$ is called the classical Euler polynomials, cf. [2, 5].

Remark 5 Taking $u=-1$ and $\lambda=1$ in Definition 2, one can see

$$
H(x,-1,1):=\frac{G_{n+1}(x)}{n+1}
$$

where $G_{n}(x)$ is called the classical Genocchi polynomials, cf. [2, 5].

Proposition 1 The following identity holds true:

$$
\lambda H_{n}(x+1, u, \lambda)-u H_{n}(x, u, \lambda)=(1-u) x^{n}, \quad c f .[5] .
$$

Proof It is proved by using Definition 2 as follows:

$$
\begin{aligned}
\sum_{n=0}^{\infty}\left(\lambda H_{n}(x+1, u, \lambda)-u H_{n}(x, u, \lambda)\right) \frac{t^{n}}{n !} & =\lambda \frac{1-u}{\lambda e^{t}-u} e^{(x+1) t}-u \frac{1-u}{\lambda e^{t}-u} e^{x t} \\
& =(1-u) e^{x t} \\
& =\sum_{n=0}^{\infty}(1-u) x^{n} \frac{t^{n}}{n !} .
\end{aligned}
$$

Matching the coefficients $\frac{t^{n}}{n !}$ gives the required result.

Proposition 2 The following identity holds true:

$$
\frac{d}{d x} H_{n}(x, u, \lambda)=n H_{n-1}(x, u, \lambda), \quad c f .[5] .
$$

Proof From Definition 2, we have

$$
\begin{aligned}
\frac{d}{d x}\left(\sum_{n=0}^{\infty} H_{n}(x, u, \lambda) \frac{t^{n}}{n !}\right) & =\sum_{n=0}^{\infty}\left(\frac{d}{d x} H_{n}(x, u, \lambda)\right) \frac{t^{n}}{n !} \\
& =\frac{d}{d x}\left(\frac{1-u}{\lambda e^{t}-u} e^{x t}\right) \\
& =\frac{1-u}{\lambda e^{t}-u} t e^{x t} \\
& =\sum_{n=0}^{\infty} H_{n}(x, u, \lambda) \frac{t^{n+1}}{n !} .
\end{aligned}
$$

Comparing the coefficients $t^{n}$ yields the desired result.

Proposition 3 Let $n$ be a member of the natural numbers. Then we have

$$
\int_{0}^{1} H_{n}(x, u, \lambda) d x=\frac{u-\lambda}{\lambda} \frac{H_{n+1}(u, \lambda)}{n+1}, \quad c f .[5] .
$$


Proof From Definition 2 and Proposition 1, we have

$$
\begin{aligned}
\int_{0}^{1} H_{n}(x, u, \lambda) d x & =\frac{H_{n+1}(1, u, \lambda)-H_{n+1}(u, \lambda)}{n+1} \\
& =\frac{u-\lambda}{\lambda} \frac{H_{n+1}(u, \lambda)}{n+1} .
\end{aligned}
$$

Thus we complete the proof of the theorem.

We are now in a position to state our main results in the next section. Also we derive their special cases.

\section{Main results}

We begin with the following theorem, which is a Fourier series expansion of the Apostol Frobenius-Euler polynomial. For the following theorem, we will give two proofs. The first proof includes the Cauchy residue theorem and a complex integral over a circle $C$ following Bayad's method in [4]. The second proof includes the Lipschitz summation formula following Luo's method in [3].

Theorem 1 Let $u, \lambda \in \mathbb{C}$ with $u \neq 1, \lambda \neq 1, u \neq \lambda$ and $0<x<1$. We have

$$
H_{n}(x, u, \lambda)=\frac{u-1}{u} n !\left(\frac{u}{\lambda}\right)^{x} \sum_{k \in \mathbb{Z}} \frac{e^{2 k \pi i x}}{\left(2 \pi i k-\log \left(\frac{\lambda}{u}\right)\right)^{n+1}} .
$$

Proof 1 of Theorem 1 We firstly consider the following integral and the function $f_{n}(t)=$ $\frac{1}{t^{n+1}} \frac{1-u}{\lambda e^{t}-u} e^{x t}:$

$$
\int_{C} f_{n}(t) d t
$$

over the circle $C=\left\{t|| t \mid \leq(2 N+\varepsilon) \pi\right.$ and $\left.\varepsilon \in \mathbb{R},\left(\varepsilon \pi i \pm \log \left(\frac{\lambda}{u}\right) \neq 0(\bmod 2 \pi i)\right)\right\}$. Now we find the poles of the function $f_{n}(t)$ as follows:

$$
t_{k}=2 k \pi i-\log \left(\frac{\lambda}{u}\right) \quad(k \in \mathbb{Z})
$$

and $t=0$ is a pole of order $n+1$. From the Cauchy residue theorem, we write

$$
\int_{C} f_{n}(t) d t=2 \pi i\left(\operatorname{Res}\left(f_{n}(t), t=0\right)+\sum_{k \in \mathbb{Z}} \operatorname{Res}\left(f_{n}(t), t=t_{k}\right)\right) .
$$

We should compute $\operatorname{Res}\left(f_{n}(t), t=0\right)$ and $\operatorname{Res}\left(f_{n}(t), t=t_{k}\right)$ as follows:

$$
\begin{aligned}
\operatorname{Res}\left(f_{n}(t), t=0\right) & =\lim _{t \rightarrow 0} \frac{1}{n !} \frac{d^{n}}{d t^{n}}(t-0)^{n+1} \frac{1}{t^{n+1}} \sum_{m=0}^{\infty} H_{m}(x, u, \lambda) \frac{t^{m}}{m !} \\
& =\lim _{t \rightarrow 0} \frac{1}{n !} \frac{d^{n}}{d t^{n}} \sum_{m=0}^{\infty} H_{m}(x, u, \lambda) \frac{t^{m}}{m !}
\end{aligned}
$$




$$
\begin{aligned}
& =\lim _{t \rightarrow 0} \frac{1}{n !} \sum_{m=0}^{\infty} H_{m}(x, u, \lambda) \frac{t^{m-n}}{(m-n) !} \\
& =\frac{H_{n}(x, u, \lambda)}{n !}
\end{aligned}
$$

and

$$
\begin{aligned}
\operatorname{Res}\left(f_{n}(t), t=t_{k}\right) & =\lim _{t \rightarrow t_{k}}\left(t-t_{k}\right) t^{-(n+1)} \frac{1-u}{\lambda e^{t}-u} e^{x t} \\
& =\frac{1}{t_{k}^{n+1}}(1-u) e^{x t_{k}} \lim _{t \rightarrow t_{k}} \frac{t-t_{k}}{\lambda e^{t}-u} \\
& =\frac{1}{t_{k}^{n+1}}(1-u) e^{x t_{k}} \lim _{t \rightarrow t_{k}} \frac{1}{\lambda e^{t}} \\
& =\frac{1}{t_{k}^{n+1}}(1-u) e^{x t_{k}} \frac{1}{\lambda e^{t_{k}}} \\
& =\frac{1}{\left(2 k \pi i-\log \left(\frac{\lambda}{u}\right)\right)^{n+1}}(1-u) e^{x\left(2 k \pi i-\log \left(\frac{\lambda}{u}\right)\right)} \frac{1}{\lambda e^{2 k \pi i-\log \left(\frac{\lambda}{u}\right)}} \\
& =\frac{1-u}{u}\left(\frac{\lambda}{u}\right)^{-x}\left(2 k \pi i-\log \left(\frac{\lambda}{u}\right)\right)^{-n-1} e^{2 k x \pi i} \cdot
\end{aligned}
$$

Combining these residues with Eq. (5) yields

$$
\int_{C} f_{n}(t) d t=2 \pi i\left(\frac{H_{n}(x, u, \lambda)}{n !}+\frac{1-u}{u}\left(\frac{u}{\lambda}\right)^{x} \sum_{k \in \mathbb{Z}} \frac{e^{2 k x \pi i}}{\left(2 k \pi i-\log \left(\frac{\lambda}{u}\right)\right)^{n+1}}\right) .
$$

From this, taking $N \rightarrow \infty$, it becomes $\int_{C} f_{n}(t) d t=0$. So we have

$$
H_{n}(x, u, \lambda)=\frac{u-1}{u} n !\left(\frac{u}{\lambda}\right)^{x} \sum_{k \in \mathbb{Z}} \frac{e^{2 k x \pi i}}{\left(2 k \pi i-\log \left(\frac{\lambda}{u}\right)\right)^{n+1}} .
$$

Therefore, we complete the proof.

Before giving the second proof of Theorem 1, we need the following definition.

Definition 4 Lipschitz summation formula is defined by

$$
\sum_{k+\mu>0} \frac{e^{2 \pi i \tau(k+\mu)}}{(k+\mu)^{1-\alpha}}=\frac{\Gamma(\alpha)}{(-2 \pi i)^{\alpha}} \sum_{k \in \mathbb{Z}} \frac{e^{-2 \pi i k \mu}}{(\tau+k)^{\alpha}} \quad(\alpha \in \mathbb{C}),
$$

where $\mu \in \mathbb{Z}, \operatorname{Re}(\alpha)>1$ if $\mu \in \mathbb{R} \backslash \mathbb{Z}, \operatorname{Re}(\alpha)>0, \tau \in H, H$ denotes the complex upper half plane; $\Gamma$ denotes the Euler-Gamma function; cf. [3].

Proof 2 of Theorem 1 By writing $t=2 \pi i \tau$ in Definition 2, we have

$$
\begin{aligned}
\sum_{k=0}^{\infty} H_{k}(x, u, \lambda) \frac{(2 \pi i \tau)^{k}}{k !} & =\frac{1-u}{\lambda e^{2 \pi i \tau}-u} e^{2 \pi i x \tau} \\
& =(u-1) e^{2 \pi i x \tau} \frac{1}{u-\lambda e^{2 \pi i \tau}}
\end{aligned}
$$




$$
\begin{aligned}
& =\frac{u-1}{u} e^{2 x \pi i \tau} \frac{1}{1-\frac{\lambda}{u} e^{2 \pi i \tau}} \quad\left(\left|\frac{\lambda}{u}\right|<1\right) \\
& =\frac{u-1}{u} \sum_{k=0}^{\infty}\left(\frac{\lambda}{u}\right)^{k} e^{2 \pi i(k+x) \tau} .
\end{aligned}
$$

Differentiating $n$ times with respect to $\tau$ gives

$$
\sum_{k=0}^{\infty} H_{k}(x, u, \lambda)(2 \pi i)^{k} \frac{\tau^{k-n}}{(k-n) !}=(2 \pi i)^{n} \frac{u-1}{u} \sum_{k=0}^{\infty}\left(\frac{\lambda}{u}\right)^{k}(k+x)^{n} e^{2 \pi i(k+x) \tau} .
$$

From Definition 4 , if we substitute $\alpha=n+1, \mu=x$ and if $\tau$ is replaced by $\tau+\log \left(\frac{\lambda}{u}\right)$, we derive

$$
\begin{aligned}
\sum_{k+x>0} \frac{e^{2 \pi i(k+x)\left(\tau+\log \left(\frac{\lambda}{u}\right)\right)}}{(k+x)^{-n}} & =\frac{n !}{(-2 \pi i)^{n+1}} \sum_{k \in \mathbb{Z}} \frac{e^{-2 \pi i k x}}{\left(\tau+\frac{\log \left(\frac{\lambda}{u}\right)}{2 \pi i}+k\right)^{n+1}} \\
& =(-1)^{n+1} n ! \sum_{k \in \mathbb{Z}} \frac{e^{-2 \pi i k x}}{\left(2 \pi i(\tau+k)+\log \left(\frac{\lambda}{u}\right)\right)^{n+1}} .
\end{aligned}
$$

From this, we reach the following expression:

$$
\left(\frac{\lambda}{u}\right)^{x} \sum_{k=0}^{\infty}(k+x)^{n}\left(\frac{\lambda}{u}\right)^{k} e^{2 \pi i(k+x) \tau}=(-1)^{n+1} n ! \sum_{k \in \mathbb{Z}} \frac{e^{-2 \pi i k x}}{\left(2 \pi i(\tau+k)+\log \left(\frac{\lambda}{u}\right)\right)^{n+1}}
$$

By (6) and (7), we see that

$$
\begin{aligned}
& \sum_{k=0}^{\infty} H_{k}(x, u, \lambda)(2 \pi i)^{k} \frac{\tau^{k-n}}{(k-n) !} \\
& \quad=(2 \pi i)^{n} \frac{u-1}{u} \sum_{k=0}^{\infty}(k+x)^{n}\left(\frac{\lambda}{u}\right)^{k} e^{2 \pi i(k+x) \tau} \\
& \quad=(2 \pi i)^{n} \frac{u-1}{u}\left(\frac{\lambda}{u}\right)^{-x}(-1)^{n+1} n ! \sum_{k \in \mathbb{Z}} \frac{e^{-2 \pi i k x}}{\left(2 \pi i(\tau+k)+\log \left(\frac{\lambda}{u}\right)\right)^{n+1}} .
\end{aligned}
$$

Taking $\tau \rightarrow 0$ in (8), we arrive at

$$
H_{n}(x, u, \lambda)=\frac{u-1}{u}\left(\frac{u}{\lambda}\right)^{x} n ! \sum_{k \in \mathbb{Z}} \frac{e^{2 \pi i k x}}{\left(2 \pi i k-\log \left(\frac{\lambda}{u}\right)\right)^{n+1}}
$$

which is the desired result.

Corollary 2 From Remark 4 and Theorem 1, we have

$$
E_{n}(x)=\frac{2 n !}{(2 \pi i)^{n+1}} \sum_{k \in \mathbb{Z}} \frac{e^{2 \pi i\left(k+\frac{1}{2}\right) x}}{\left(k+\frac{1}{2}\right)^{n+1}}, \quad c f .[3,4] .
$$


Proof It follows from Remark 4 and Theorem 1 that

$$
H_{n}(x,-1,1):=E_{n}(x)=2(-1)^{x} n ! \sum_{k \in \mathbb{Z}} \frac{e^{2 \pi i k x}}{(2 \pi i k-\log (-1))^{n+1}} .
$$

Since

$$
(-1)^{x}:=e^{-\pi i x} \text { and } \log (-1)=-\pi i
$$

with the logarithmic property over the complex plane,

$$
z=|z| e^{i \theta}, \quad-\pi \leq \theta<\pi \quad \text { and } \quad \log (z)=\log (|z|)+i \theta
$$

we can write

$$
\begin{aligned}
E_{n}(x) & =2 n ! \sum_{k \in \mathbb{Z}} \frac{e^{2 \pi i k x-\pi i x}}{(2 \pi i k+\pi i)^{n+1}} \\
& =\frac{2 n !}{(2 \pi i)^{n+1}} \sum_{k \in \mathbb{Z}} \frac{e^{2 \pi i\left(k-\frac{1}{2}\right)}}{\left(k+\frac{1}{2}\right)^{n+1}},
\end{aligned}
$$

which completes the proof of this corollary.

Corollary 3 By making use of the relation $H(x,-1,1):=\frac{G_{n+1}(x)}{n+1}$ in Theorem 1 , we have

$$
G_{n}(x)=\frac{2 n !}{(2 \pi i)^{n}} \sum_{k \in \mathbb{Z}} \frac{e^{2 \pi i\left(k+\frac{1}{2}\right) x}}{\left(k+\frac{1}{2}\right)^{n}}, \quad c f .[4] .
$$

Corollary 4 Putting $\lambda=1$ in Theorem 1 we have

$$
H_{n}(x, u)=\frac{u-1}{u} n ! u^{x} \sum_{k \in \mathbb{Z}} \frac{e^{2 k x \pi i}}{(2 k \pi i-\log (u))^{n+1}} .
$$

Corollary 5 Substituting $u=-1$ in Theorem 1 yields

$$
E_{n}(x, \lambda)=\frac{2 n !}{\lambda^{x}} \sum_{k \in \mathbb{Z}} \frac{e^{2 \pi i\left(k-\frac{1}{2}\right) x}}{(2 k \pi i-\pi i-\log (\lambda))^{n+1}}, \quad c f .[3,4] .
$$

From a Fourier expanison of the Apostol Frobenius-Euler polynomials, we derive the following interesting identity.

Theorem 2 Let L be a positive integer. Then we have

$$
\begin{aligned}
& \frac{1}{L} \sum_{j=0}^{L-1}\left(\frac{\lambda}{u}\right)^{\frac{j}{L}} H_{n}\left(\frac{x+j}{L}, u, \lambda\right) \\
& \quad=H_{n}\left(\frac{x}{L}, u, \lambda\right)+\frac{1}{L} \frac{u-1}{u}\left(\frac{u}{\lambda}\right)^{x} n ! \sum_{k \in \mathbb{Z}}\left(\sum_{\substack{j=0 \\
k \neq 0(\bmod L)}}^{L-1} \frac{e^{2 \pi i k\left(\frac{x+j}{L}\right)}}{\left(2 \pi i k-\log \left(\frac{\lambda}{u}\right)\right)^{n+1}}\right) .
\end{aligned}
$$


Proof From Theorem 1, we derive the following applications:

$$
\begin{aligned}
& \sum_{j=0}^{L-1}\left(\frac{\lambda}{u}\right)^{\frac{j}{L}} H_{n}\left(\frac{x+j}{L}, u, \lambda\right) \\
& =\sum_{j=0}^{L-1}\left(\frac{\lambda}{u}\right)^{\frac{j}{L}}\left(\frac{u-1}{u} n !\left(\frac{u}{\lambda}\right)^{\frac{x+j}{L}} \sum_{k \in \mathbb{Z}} \frac{e^{2 \pi i\left(\frac{x+j}{L}\right)}}{\left(2 \pi i k-\log \left(\frac{\lambda}{u}\right)\right)^{n+1}}\right) \\
& =\frac{u-1}{u}\left(\frac{u}{\lambda}\right)^{\frac{x}{L}} n ! \sum_{k \in \mathbb{Z}}\left(\sum_{\substack{j=0 \\
k \equiv 0(\bmod L)}}^{L-1} \frac{e^{2 k \pi i\left(\frac{x+j}{L}\right)}}{\left(2 \pi i k-\log \left(\frac{\lambda}{u}\right)\right)^{n+1}}+\sum_{\substack{j=0 \\
k \neq 0(\bmod L)}}^{L-1} \frac{e^{2 k \pi i\left(\frac{x+j}{L}\right)}}{\left(2 \pi i k-\log \left(\frac{\lambda}{u}\right)\right)^{n+1}}\right) .
\end{aligned}
$$

Under the following condition:

$$
\sum_{j=0}^{L-1} e^{2 \pi i j \frac{k}{L}}=L \quad(k \equiv 0(\bmod L))
$$

we have

$$
\begin{aligned}
& =\frac{u-1}{u}\left(\frac{u}{\lambda}\right)^{\frac{x}{L}} n ! \sum_{k \in \mathbb{Z}}\left(\frac{L e^{2 k \pi i \frac{x}{L}}}{\left(2 \pi i k-\log \left(\frac{\lambda}{u}\right)\right)^{n+1}}+\sum_{\substack{j=0 \\
k \neq 0(\bmod L)}}^{L-1} \frac{e^{2 k \pi i\left(\frac{x+j}{L}\right)}}{\left(2 \pi i k-\log \left(\frac{\lambda}{u}\right)\right)^{n+1}}\right) \\
& =L H_{n}\left(\frac{x}{L}, u, \lambda\right)+\frac{u-1}{u}\left(\frac{u}{\lambda}\right)^{\frac{x}{L}} n ! \sum_{k \in \mathbb{Z}}\left(\sum_{\substack{j=0 \\
k \neq 0(\bmod L)}}^{L-1} \frac{e^{2 k \pi i\left(\frac{x+j}{L}\right)}}{\left(2 \pi i k-\log \left(\frac{\lambda}{u}\right)\right)^{n+1}}\right) .
\end{aligned}
$$

Thus we complete the proof of the theorem.

Theorem 3 Let $0<x<1$. We have

$$
H_{n}(x, u, \lambda)=\sum_{k \in \mathbb{Z}} c_{k, n} e^{2 k \pi i x}
$$

with the following the coefficients $c_{k, n}$ :

$$
c_{k, n}=\frac{\lambda-u}{\lambda} \sum_{l=1}^{n-2} \frac{(n)_{l} H_{n-l+1}(u, \lambda)}{(2 \pi i k)^{l}(n-l+1)}+\frac{u-1}{\lambda-u} \frac{n !}{(2 \pi i k)^{n}},
$$

where $(n)_{l}$ is the falling factorial.

Proof Let

$$
H_{n}(x, u, \lambda)=\sum_{k \in \mathbb{Z}} c_{k, n} e^{2 k \pi i x}
$$


with the following coefficients:

$$
c_{k, n}=\int_{0}^{1} H_{n}(x, u, \lambda) e^{-2 \pi i k x} d x
$$

By integration by parts, we have

$$
\begin{aligned}
c_{k, n} & =\frac{u-\lambda}{\lambda} \frac{H_{n+1}(u, \lambda)}{n+1}+\frac{2 \pi i k}{n+1} \int_{0}^{1} H_{n+1}(x, u, \lambda) e^{-2 \pi i k x} d x \\
& =\frac{u-\lambda}{\lambda} \frac{H_{n+1}(u, \lambda)}{n+1}+\frac{2 \pi i k}{n+1} c_{k, n+1} .
\end{aligned}
$$

From this, we find the following recurrence relation:

$$
c_{k, n}=\frac{n}{2 \pi i k} \frac{\lambda-u}{\lambda} \frac{H_{n}(u, \lambda)}{n}+\frac{n}{2 \pi i k} c_{k, n-1} .
$$

By the iteration method, we arrive at the following expression:

$$
c_{k, n}=\frac{\lambda-u}{\lambda} \sum_{l=1}^{n-2} \frac{(n)_{l} H_{n-l+1}(u, \lambda)}{(2 \pi i k)^{l}(n-l+1)}+\frac{n !}{(2 \pi i k)^{n-1}} c_{k, 1} .
$$

Now it seems to be sufficient in order to compute $c_{k, 1}$. Since

$$
H_{1}(x, u, \lambda)=\frac{1-u}{\lambda-u} x-\frac{\lambda(1-u)}{(\lambda-u)^{2}},
$$

we have

$$
\begin{aligned}
c_{k, 1} & =\int_{0}^{1}\left(\frac{1-u}{\lambda-u} x-\frac{\lambda(1-u)}{(\lambda-u)^{2}}\right) e^{-2 \pi i k x} d x \\
& =\frac{u-1}{\lambda-u} \frac{1}{2 \pi i k} .
\end{aligned}
$$

Also

$$
c_{k, n}=\frac{\lambda-u}{\lambda} \sum_{l=1}^{n-2} \frac{(n)_{l} H_{n-l+1}(u, \lambda)}{(2 \pi i k)^{l}(n-l+1)}+\frac{1-u}{\lambda-u} \frac{n !}{(2 \pi i k)^{n}} .
$$

Thus we end the proof.

In [9], Kim et al. defined the Hurwitz type $\lambda$-zeta function as follows:

$$
\zeta_{\lambda}(s, x)=\sum_{n=0}^{\infty} \frac{\lambda^{n}}{(n+x)^{s}} \quad(s \in \mathbb{C})
$$

Note that $\zeta_{\lambda}(s, x)$ when $\lambda=-1$ is the Hurwitz-Euler zeta function; cf. [10].

Recall from Eq. (8) that

$$
\sum_{k=0}^{\infty} H_{k}(x, u, \lambda) \frac{t^{n}}{n !}=\frac{u-1}{u} \sum_{k=0}^{\infty}\left(\frac{\lambda}{u}\right)^{k} e^{(k+x) t} \quad\left(\left|\frac{\lambda}{u} e^{t}\right|<1\right) .
$$


From this we have

$$
H_{n}(x, u, \lambda)=\frac{u-1}{u} \sum_{k=0}^{\infty}\left(\frac{\lambda}{u}\right)^{k}(k+x)^{n} .
$$

Then Eq. (9) can be written

$$
\begin{aligned}
\frac{u-1}{u} \zeta_{\frac{\lambda}{u}}(-n, x) & =\frac{u-1}{u} \sum_{k=0}^{\infty}(k+x)^{n}\left(\frac{\lambda}{u}\right)^{k} \\
& =H_{n}(x, u, \lambda) .
\end{aligned}
$$

Thus we have the following theorem.

Theorem 4 The following equality holds true:

$$
\frac{u-1}{u} \zeta_{\frac{\lambda}{u}}(-n, x)=H_{n}(x, u, \lambda)
$$

In [9], Kim et al. introduced the $\lambda$-partial zeta function as follows:

$$
\mathbf{H}_{\lambda}(s, a \mid F)=\sum_{m \equiv a(\bmod F)} \frac{\lambda^{m}}{m^{s}}
$$

From this we have the following applications:

$$
\begin{aligned}
\mathbf{H}_{\frac{\lambda}{u}}(s, a \mid F) & =\sum_{m=0}^{\infty} \frac{\left(\frac{\lambda}{u}\right)^{m F+a}}{(m F+a)^{s}} \\
& =\lambda^{a} F^{-s} \sum_{m=0}^{\infty} \frac{\left(\left(\frac{\lambda}{u}\right)^{F}\right)^{m}}{\left(m+\frac{a}{F}\right)^{s}} \\
& =\lambda^{a} F^{-s} \zeta_{\frac{\lambda^{F}}{u^{F}}}\left(s, \frac{a}{F}\right) .
\end{aligned}
$$

By Theorem 4 and Eq. (10), we have the following theorem.

Theorem 5 The following identity holds true:

$$
\lambda^{a} F^{n} H_{n}\left(\frac{a}{F}, u^{F}, \lambda^{F}\right)=\frac{u^{F}-1}{u^{F}} \mathbf{H}_{\frac{\lambda}{u}}(-n, a \mid F) .
$$

Set $\lambda=e^{2 \pi i x}, x=-\frac{\log \left(\frac{\lambda}{u}\right)}{2 \pi i}$ and $s=n+1$ in Eq. (9), we see that

$$
\zeta_{e^{2 \pi i x}}\left(n+1,-\frac{\log \left(\frac{\lambda}{u}\right)}{2 \pi i}\right)=\sum_{k=0}^{\infty} \frac{e^{2 \pi i k x}}{\left(k-\frac{\log \left(\frac{\lambda}{u}\right)}{2 \pi i}\right)^{n+1}} .
$$


Now we write the Fourier expansion of the Apostol Frobenius-Euler polynomials as follows:

$$
\begin{aligned}
H_{n}(x, u, \lambda)= & \frac{u-1}{u} n !\left(\frac{u}{\lambda}\right)^{x} \frac{1}{\left(-\log \frac{\lambda}{u}\right)^{n+1}}+\frac{\frac{u-1}{u} n !\left(\frac{u}{\lambda}\right)^{x}}{(2 \pi i)^{n+1}} \sum_{k=1}^{\infty} \frac{e^{2 k \pi i x}}{\left(k-\log \left(\frac{\lambda}{u}\right)\right)^{n+1}} \\
& +\frac{\frac{u-1}{u} n !\left(\frac{u}{\lambda}\right)^{x}}{(-2 \pi i)^{n+1}} \sum_{k=1}^{\infty} \frac{e^{-2 k \pi i x}}{\left(k+\log \left(\frac{\lambda}{u}\right)\right)^{n+1}},
\end{aligned}
$$

which is closely related to Eq. (11). So we have

$$
\begin{aligned}
H_{n}(x, u, \lambda)= & \frac{u-1}{u} n !\left(\frac{u}{\lambda}\right)^{x} \frac{1}{\left(-\log \frac{\lambda}{u}\right)^{n+1}}+\frac{\frac{u-1}{u} n !\left(\frac{u}{\lambda}\right)^{x}}{(2 \pi i)^{n+1}} \zeta_{e^{2 \pi i x}}\left(n+1,-\frac{\log \left(\frac{\lambda}{u}\right)}{2 \pi i}\right) \\
& +\frac{\frac{u-1}{u} n !\left(\frac{u}{\lambda}\right)^{x}}{(2 \pi i)^{n+1}} \zeta_{e^{-2 \pi i x}}\left(n+1, \frac{\log \left(\frac{\lambda}{u}\right)}{2 \pi i}\right) .
\end{aligned}
$$

Thus we state the following theorem.

Theorem 6 Let $u, \lambda \in \mathbb{C}$ with $u \neq 1, \lambda \neq 1, u \neq \lambda$ and $0<x<1$. We have

$$
\begin{aligned}
H_{n}(x, u, \lambda)= & \frac{u-1}{u} n !\left(\frac{u}{\lambda}\right)^{x} \frac{1}{\left(-\log \frac{\lambda}{u}\right)^{n+1}}+\frac{\frac{u-1}{u} n !\left(\frac{u}{\lambda}\right)^{x}}{(2 \pi i)^{n+1}} \zeta_{e^{2 \pi i x}}\left(n+1,-\frac{\log \left(\frac{\lambda}{u}\right)}{2 \pi i}\right) \\
& +\frac{\frac{u-1}{u} n !\left(\frac{u}{\lambda}\right)^{x}}{(2 \pi i)^{n+1}} \zeta_{e^{-2 \pi i x}}\left(n+1, \frac{\log \left(\frac{\lambda}{u}\right)}{2 \pi i}\right) .
\end{aligned}
$$

\section{Further remarks}

Based on Definition 3, we introduce here the Apostol Frobenius-Genocchi polynomials $G_{n}^{F}(x, u, \lambda)$ by the following definition.

Definition 5 Let $u \in \mathbb{C}$ with $u \neq 1$. We define the Apostol Frobenius-Genocchi polynomials as follows:

$$
\sum_{n=0}^{\infty} G_{n}^{F}(x, u, \lambda) \frac{t^{n}}{n !}=\frac{(1-u) t}{\lambda e^{t}-u} e^{x t} \quad(\lambda \in \mathbb{C})
$$

The Apostol Frobenius-Genocchi polynomials are closely related to the Apostol Frobenius-Euler polynomials by the following application:

$$
\begin{aligned}
\sum_{n=0}^{\infty} G_{n}^{F}(x, u, \lambda) \frac{t^{n}}{n !} & =t \sum_{n=0}^{\infty} H_{n}(x, u, \lambda) \frac{t^{n}}{n !} \\
& =\sum_{n=0}^{\infty} H_{n}(x, u, \lambda) \frac{t^{n+1}}{n !},
\end{aligned}
$$

which gives

$$
H_{n}(x, u, \lambda)=\frac{G_{n+1}^{F}(x, u, \lambda)}{n+1} .
$$


We now give some of fundamental properties of the Apostol Frobenius-Genocchi polynomials. We will omit the proof, since it follows from Definition 5.

Theorem 7 The derivative property of the Apostol Frobenius-Genocchi polynomials is as follows:

$$
\frac{d}{d x} G_{n}^{F}(x, u, \lambda)=n G_{n-1}^{F}(x, u, \lambda)
$$

Theorem 8 Difference property of Apostol Frobenius-Genocchi polynomials is as follows:

$$
\lambda G_{n}^{F}(x+1, u, \lambda)-u G_{n}^{F}(x, u, \lambda)=(1-u) n x^{n-1} .
$$

Theorem 9 The integral of Apostol Frobenius-Genocchi polynomials from a to $b$, where $a, b$ are members of real numbers, is as follows:

$$
\int_{a}^{b} G_{n}^{F}(x, u, \lambda) d x=\frac{G_{n+1}^{F}(b, u, \lambda)-G_{n+1}^{F}(a, u, \lambda)}{n+1} .
$$

Theorem 10 For $\left|\frac{\lambda e^{t}}{u}\right|<1$, the generating function of the Apostol Frobenius-Genocchi polynomials can be written in the following form:

$$
\sum_{n=0}^{\infty} G_{n}^{F}(x, u, \lambda) \frac{t^{n}}{n !}=\frac{u-1}{u} t e^{x t} \sum_{m=0}^{\infty}\left(\frac{\lambda}{u}\right)^{m} e^{t(m+x)} .
$$

Theorem 11 By the relation $H_{n}(x, u, \lambda)=\frac{G_{n+1}^{F}(x, u, \lambda)}{n+1}$ in Theorem 1, we have

$$
G_{n}^{F}(x, u, \lambda)=\frac{u-1}{u}\left(\frac{u}{\lambda}\right)^{x} n ! \sum_{k \in \mathbb{Z}} \frac{e^{2 \pi i k x}}{\left(2 \pi i k-\log \left(\frac{\lambda}{u}\right)\right)^{n}}
$$

which represents a Fourier expansion of the Apostol Frobenius-Genocchi polynomials.

\section{Conclusion and observation}

In the paper, we have derived the Fourier expansion of Apostol Frobenius-Euler polynomials as Theorem 1. We have investigated special cases of Theorem 1 turning to Fourier expansions of Euler polynomials, Genocchi polynomials, Frobenius-Euler polynomials, Apostol-Euler polynomials, Apostol Genocchi polynomials. With the motivation of the work [12], we have introduced Apostol Frobenius-Genocchi polynomials. We saw that Apostol Frobenius-Genocchi polynomials are closely related to Apostol Frobenius-Euler polynomials by the following relation:

$$
H_{n}(x, u, \lambda)=\frac{G_{n+1}^{F}(x, u, \lambda)}{n+1} .
$$

By this relation, we have got a Fourier expansion for the Apostol Frobenius-Genocchi polynomials,

$$
G_{n}^{F}(x, u, \lambda)=\frac{u-1}{u}\left(\frac{u}{\lambda}\right)^{x} n ! \sum_{k \in \mathbb{Z}} \frac{e^{2 \pi i k x}}{\left(2 \pi i k-\log \left(\frac{\lambda}{u}\right)\right)^{n}} .
$$


By Eq. (12), the obtained theorems concerning the Apostol Frobenius-Euler polynomials here and in other sources turn into those concerning Apostol Frobenius-Genocchi polynomials.

\section{Acknowledgements}

The authors are grateful to the Editor, Prof. Dr. Taekyun Kim, and to the referees for their valuable suggestions, which have improved the paper substantially.

\section{Competing interests}

The authors declare that they have no competing interests.

Authors' contributions

All authors contributed equally to the manuscript. All authors read and approved the final manuscript.

\section{Author details}

${ }^{1}$ Department of Economics, Faculty of Economics, Administrative and Social Science, Hasan Kalyoncu University, Gaziantep, Turkey. ${ }^{2}$ Department of Mathematics, Faculty of Science and Arts, University of Gaziantep, Gaziantep, Turkey.

\section{Publisher's Note}

Springer Nature remains neutral with regard to jurisdictional claims in published maps and institutional affiliations.

Received: 22 December 2017 Accepted: 15 February 2018 Published online: 22 February 2018

References

1. Agarwal, R.P., Kim, D.S., Kim, T., Kwon, J.: Sums of finite products of Bernoulli functions. Adv. Differ. Equ. 2017, 237 (2017)

2. Kim, T.: Identities involving Frobenius-Euler polynomials arising from non-linear differential equations. J. Number Theory 132, 2854-2865 (2012)

3. Luo, Q.-M.: Fourier expansions and integral representations for the Apostol-Bernoulli and Apostol-Euler polynomials. Math. Comput. 78(268), 2193-2208 (2009)

4. Bayad, A.: Fourier expansions for Apostol-Bernoulli, Apostol-Euler and Apostol-Genocchi polynomials. Math. Comput. 80(276), 2219-2221 (2011)

5. Bayad, A., Kim, T.: Identities for Apostol-type Frobenius-Euler polynomials resulting from the study of a nonlinear operator. Russ. J. Math. Phys. 23(2), 164-171 (2016)

6. Kim, T., Kim, D.S., Jang, G.-W., Kwon, J.: Fourier series of sums of products of Genocchi functions and their applications. J. Nonlinear Sci. Appl. 10, 1683-1694 (2017)

7. Jang, G.-W., Kim, T., Kim, D.S., Mansour, T.: Fourier series of functions related to Bernoulli polynomials. Adv. Stud. Contemp. Math. 27, 49-62 (2017)

8. Kim, T., Kim, D.S., Rim, S.-H., Dolgy, D.: Fourier series of higher-order Bernoulli functions and their applications. J. Inequal. Appl. 2017, 8 (2017)

9. Kim, T., Rim, S.-H., Simsek, Y., Kim, D.: On the analogs of Bernoulli and Euler numbers, related identities and zeta and L-functions. J. Korean Math. Soc. 45(2), 435-453 (2008)

10. Kim, T.: Euler numbers and polynomials associated with zeta functions. Abstr. Appl. Anal. 2008, Article ID 581582 (2008)

11. Kim, T., Kim, D.S., Dolgy, D.V., Park, J.-W.: Fourier series of sums of products of ordered Bell and poly-Bernoulli functions. J. Inequal. Appl. 2017, 84 (2017)

12. Yilmaz, B., Ozarslan, M.A.: Frobenius-Euler and Frobenius-Genocchi polynomials and their differential equations. New Trends Math. Sci. 3, 172-180 (2015)

\section{Submit your manuscript to a SpringerOpen ${ }^{\circ}$ journal and benefit from:}

- Convenient online submission

- Rigorous peer review

- Open access: articles freely available online

- High visibility within the field

- Retaining the copyright to your article

Submit your next manuscript at $\boldsymbol{\triangleright}$ springeropen.com 